\title{
Identification and verification of HCAR3 and INSL5 as new potential therapeutic targets of colorectal cancer
}

Xuan Yang ${ }^{1 \dagger}$, Wangao Wei $^{3 \dagger}$, Shisheng Tan ${ }^{1,2}$, Linrui Guo ${ }^{3}$, Song Qiao ${ }^{3}$, Biao Yao ${ }^{3 *}$ and Zi Wang ${ }^{1,2^{*}}$

\begin{abstract}
Background: Colorectal cancer (CRC) is one of the most common cancers of the gastrointestinal tract and ranks third in cancer-related deaths worldwide. This study was conducted to identify novel biomarkers related to the pathogenesis of CRC based upon a bioinformatics analysis, and further verify the biomarkers in clinical tumor samples and CRC cell lines.

Methods: A series of bioinformatics analyses were performed using datasets from NCBI-GEO and constructed a protein-protein interaction (PPI) network. This analysis enabled the identification of Hub genes, for which the mRNA expression and overall survival of CRC patients data distribution was explored in The Cancer Genome Atlas (TCGA) colon cancer and rectal cancer (COADREAD) database. Furthermore, the differential expression of HCAR3 and INLS5 was validated in clinical tumor samples by Real-time quantitative PCR analysis, western blotting analysis, and immunohistochemistry analysis. Finally, CRC cells over-expressing INSL5 were constructed and used for CCK8, cell cycle, and cell apoptosis validation assays in vitro.
\end{abstract}

Results: A total of 286 differentially expressed genes (DEGs) were screened, including 64 genes with increased expression and 143 genes with decreased expression in 2 CRC database, from which 10 key genes were identified: CXCL1, HCAR3, CXCL6, CXCL8, CXCL2, CXCL5, PPY, SST, INSL5, and NPY1R. Among these genes, HCAR3 and INSL5 had not previously been explored and were further verified in vitro.

Conclusions: HCAR3 expression was higher in CRC tissues and associated with better overall survival of CRC patients. INSL5 expression in normal tissue was higher than that in tumor tissue and its high expression was associated with a better prognosis for CRC. The overexpression of INSL5 significantly inhibited the proliferation and promoted the shearing of PARP of CRC cells. This integrated bioinformatics study presented 10 key hub genes associated with CRC. HCAR3 and INSL5 were expressed in tumor tissue and these were associated with poor survival and warrant further studies as potential therapeutic targets.

Keywords: Colorectal cancer, Bioinformatics, Biomarkers, HCAR3, INSL5

*Correspondence: ybb223@163.com;wz522321@163.com

†Xuan Yang and Wangao Wei contributed equally to this work.

2 Department of Oncology, Guizhou Provincial People's Hospital, Guizhou

550002 Guiyang, China

${ }^{3}$ Tongren Municipal People's Hospital, Guizhou 554300 Tongren, China

Full list of author information is available at the end of the article

\section{Introduction}

Colorectal cancer $(\mathrm{CRC})$ incidence and mortality have been consistently increasing year-over-year. The 2020 GLOBOCAN statistics show that the incidence of CRC makes it the third most common form of cancer worldwide and it has the second highest mortality rate [1]. The prognosis of CRC is much better if diagnosed at earlier stages. Based upon aggregate data, a statistical analysis original author(s) and the source, provide a link to the Creative Commons licence, and indicate if changes were made. The images or other third party material in this article are included in the article's Creative Commons licence, unless indicated otherwise in a credit line to the material. If material is not included in the article's Creative Commons licence and your intended use is not permitted by statutory regulation or exceeds the permitted use, you will need to obtain permission directly from the copyright holder. To view a copy of this licence, visit http://creativecommons.org/licenses/by/4.0/. The Creative Commons Public Domain Dedication waiver (http://creativeco mmons.org/publicdomain/zero/1.0/) applies to the data made available in this article, unless otherwise stated in a credit line to the data. 
showed that the 5-year survival rate was over $90 \%$ for patients with stage I CRC compared to about $10 \%$ for patients with stage IV CRC [2]. Treatments for CRC include surgery, peri-operative radiotherapy plus chemotherapy, targeted therapy, and immunotherapy. Because of advancements in understanding colorectal carcinogenesis, the array of treatment options for local and advanced CRC have increased and individual treatment plans have also been developed [3]. The average survival time of advanced CRC has significantly improved in the past decade $[4,5]$. However, survival is still poor for those with metastasized CRC. Thus, a further understanding of CRC pathogenesis and the identification of more prognostic markers are required.

Bioinformatics, one of the newest interdisciplinary fields incorporating biological research and computer science, has been widely used to exploring genetic correlations in tumors [6]. Recent studies have focused upon identifying core genes involved in pathogenesis and effective candidates for targeted therapy of CRC have been selected. Wang et al. identified a total of 202 differentially expressed genes (DEGs), including 58 genes with increased expression and 144 with decreased expression in CRC samples compared to normal tissues. A total of 10 hub genes were identified by cyto-Hubba: PYY, CXCL3, CXCL11, CXCL8, CXCL12, CCL20, MMP3, P2RY14, NPY1R, and CXCL1 [7]. Xu et al. found a total of 87 DEGs, including 19 genes with increased expression and 68 with decreased expression, and showed that SST, CXCL8, and MS4A12 were significantly differentially expressed between colorectal cancer tissues and normal tissues validated with RT-PCR assays [8]. Zhou et al. indicated that three novel genes: CNTN3, SLC1A1, and $S L C 16 A 9$, which had diagnostic values with respect to occurrences of colorectal cancer, from a total of 237 identified differentially expressed genes [9]. Some CRCrelated key mRNAs were also identified that increased understanding of molecular mechanisms underlying CRC development [10]. A bioinformatics approach has also been used in colonic adenoma, CRC metastases, and normal colonic epithelium samples. A total of 438 genes were identified to be differentially expressed in colonic adenoma, 885 in carcinoma, and 736 in metastases [11].

However, these biomarkers have not been sufficiently explored for potential clinical applications; largely because the aforementioned studies did not verify the identified genes in cell lines or CRC samples using molecular biological techniques. Accordingly, this study was conducted to identify and verify biomarkers using a novel data combination analysis for diagnosing and exploring the pathogenesis for new CRC therapeutic targets, and further verify them in clinical tumor samples and CRC cell lines.

\section{Materials and methods}

\section{Patients and specimens}

We retrospectively collected CRC tissues, adjacent nontumor tissues, and paraffin-embedded specimens (except for the sample 2 missed the paraffin-embedded specimens) from 5 CRC patients (including 3 males and 2 females) under-going curative resection at the Tongren Municipal People's Hospital from June 2020. Patients did not receive any preoperative anticancer treatment, including chemotherapy or radiotherapy. The mean age of these patients at the time of diagnosis was $66.8 \pm 11.1$ (range 46-77 years). The use of human samples for this project was approved by the ethics committee of Tongren Municipal People's Hospital and written informed consent was obtained from each patient.

\section{Microarray data}

The gene expression profiles from GSE9348 and GSE11024 were downloaded from GEO (https://www. ncbi.nlm.nih.gov/geo/) database. The GSE9348 data was generated using GPL570, [HG-U133_Plus_2] Affymetrix Human Genome U133 Plus 2 Array. Tumors from age and ethnicity matched 70 patients and biopsies from 12 healthy controls were used. The GSE110224 data was generated using GPL570, [HG-U133_Plus_2] Affymetrix Human Genome U133 Plus 2 Array, which consists of 17 normal colon tissue specimens and 17 tumor colon tissue specimens. The $\mathrm{R}$ software package was used to process the downloaded files and to convert and reject the unqualified data. The data was calibrated, standardized, and $\log 2$ transformed.

\section{Screening for DEGs}

The ID corresponding to the probe name was converted into an international standard name for genes (gene symbol). The differential gene expression analysis was performed using the limma package in the Bio-conductor package (http://www.bioconductor.org/). The DEGs in colorectal cancer and normal colorectal samples from two microarray datasets were considered to have a corrected $P$ value of $<0.05$ and $\mid \log 2$ fold change $(\mathrm{FC})>2 \mid$. Additional graphics packages were produced to visualize the number of differentially expressed genes (DEGs) and heatmaps. A list of genes that an increased or decreased expression in both the microarray datasets was used for the subsequent analyses.

\section{Functional enrichment analysis}

The enrichment analysis for Gene Ontology (GO) terms and Kyoto Encyclopedia of Genes and Genomes (KEGG) pathways was conducted using the R/Bioconductor package Cluster Profiler (http://www.bioconductor.org/packa ges/release/bioc/html/clusterProfiler.html) package, 
which was also used to plot the data. Only the GO terms and pathways with an adjusted $p$ value $<0.05$ were considered statistically significant..

\section{PPI network integration}

To generate an interaction network, we uploaded common different gene identifiers to the STRING database (http://string-db.org/). The STRING database is a software system that is commonly used to identify the interactions between known/predicted proteins. Additionally, Cytoscape (www.cytoscape.org/) software was used to further expand upon the obtained interaction network. Each node is a gene, protein, or molecule, and the connections between the nodes represent the interactions between the molecules. This network was used to identify interactions and pathway relationships between the proteins encoded by the DEGs in CRC. The corresponding proteins in the central node are possible core proteins or key candidate genes with potentially important regulatory or physiologically relevant functions.

\section{The expression levels and survival analysis of Hub Genes in CRC}

The mRNA expression of the hub genes and the overall survival of CRC patients were examined by data mining using The Cancer Genome Atlas (TCGA) Colon and Rectal Cancer (COADREAD) using the University of California, Santa Cruz (UCSC) Xena browser (https://ucscx enabrowser.net). The gene expression analysis was performed together with the computation of the associated box plots in $\mathrm{R}$ using the ggstatsplot (https://indrajeetp atil.github.io/ggstatsplot/). Survival curves were plotted using the Kaplan-Meier method and compared using log-rank test by R "survival" (https://github.com/thern eau/survival) package. $P$ values of $<0.05$ were considered statistically significant.

\section{Cell culture}

CRC cell lines (Stem Cell Bank, Chinese Academy of Sciences, CN) were maintained in McCoy's 5A Medium (Gibco, USA) with 10\% fetal bovine serum (NATOCOR, AR), $100 \mathrm{U} / \mathrm{ml}$ penicillin and $100 \mathrm{U} / \mathrm{ml}$ streptomycin. All cultures were maintained at $37{ }^{\circ} \mathrm{C}$ in a humidified atmosphere of $95 \%$ air and $5 \% \mathrm{CO}_{2}$. INSL5 cDNA was subcloned into the MSCV-IRES-mCherry-T2A-Puro retroviral vector. The retrovirus were produced using calcium phosphate-mediated transfection to produce stably transduced cells, which were then selected with $1 \mu \mathrm{g} / \mathrm{ml}$ puromycin (Meilun, $\mathrm{CN}$ ) prior to sorting for mCherry fluorescent protein expression.

\section{CCK-8 assay}

A CCK- 8 assay kit (Targetmol, USA) was used to measure the proliferation of HT29 and SW620 cells. A total of 5000 cells in a volume of $100 \mu \mathrm{L}$ per well in five replicate wells were cultured in a 96-well plate in medium containing $10 \%$ FBS. Then, the CCK-8 reagent $(10 \mu \mathrm{L})$ was added to McCoy's $5 \mathrm{~A}$ Medium to generate a working solution, for which $100 \mu \mathrm{L}$ was added per well and incubated for $2 \mathrm{~h}$. A microplate reader was used to detect the absorbency at a wavelength of $450 \mathrm{~nm}$.

\section{Cell cycle}

Cells were treated with $20 \mu \mathrm{M}$ BrdU (Sigma, DE) $48 \mathrm{~h}$ after seeding; $2 \mathrm{~h}$ later, the cells were harvested by centrifugation, followed by fixing with $0.4 \%$ formaldehyde for $20 \mathrm{~min}$ and washing with PBS. The cells were then fixed in $70 \%$ ethanol at $-20{ }^{\circ} \mathrm{C}$ for $12 \mathrm{~h}$. The cells were permeabilized with $2 \mathrm{~N} \mathrm{HCL} / 0.5 \%$ Triton X-100 for $20 \mathrm{~min}$ and neutralized with $0.1 \mathrm{M}$ sodium borate for 5 min prior to incubation with the anti-BrdU antibody (BD Biosciences, USA) for 20 min. Finally, the cells were resuspended in $500 \mu \mathrm{l}$ with $10 \mu \mathrm{g}$ RNase A for $30 \mathrm{~min}$. The samples were counterstained with $2 \mu \mathrm{l}$ Propidium Iodide (PI) for $15 \mathrm{~min}$. The cell cycle distribution was analyzed by CytoFLEX S (Beckman, USA) and analyzed with the Flow Jo 10.0.5 (BD, USA).

\section{Cell apoptosis}

Based upon the results of the CCK- 8 assay, the SW620 cells were used for the Annexin V-FITC/PI cell apoptosis assay (BD Biosciences, USA). SW620 cells were seeded in 6 -well plates at a density of $2 \times 10^{5}$ cells/ well. After $48 \mathrm{~h}$, the cells were harvested, washed with PBS, and stained with the Annexin V/(PI) Apoptosis Detection Kit. The fluorescence of the cells was visualized using flow cytometry, for which the simultaneous detection of apoptosis pathway related protein levels was conducted.

\section{Real-time quantitative PCR (RT-PCR) analysis}

Total RNA was extracted from patient tissue/cells with TRIzol Reagent (Invitrogen, USA). The total cellular RNA was reverse transcribed using an Evo M-MLV RT Kit with a gDNA Clean for qPCR kit (Accurate, CN). Blank reactions with no RNA were performed for all the experiments. The mRNA expression was measured by real-time PCR using SYBR Green Premix Pro Taq HS qPCR Kit (Accurate, CN). The CFX Connect TM Real-Time System (Bio-Rad, CA, USA) was used to run the RT-PCR reactions. The primer sets were as shown 
in Table S1. The relative gene expression to $\beta$-actin was calculated using the $2^{-\Delta \Delta \mathrm{CT}}$ method.

\section{Western blotting analysis}

The protein levels for the molecules of interest were determined by western blotting according to the manufacturer's protocol. The following antibodies were used: rabbit anti-HM74 (ER63689, HuaAn, CN), rabbit anti-INSL5 (ER60301, HuaAn, CN), rabbit antiBcl-2 (ET1702, HuaAn, CN), mouse anti-P21 (RT1449, HuaAn, CN), rabbit anti-Cleaved PARP (5625S, Cell signaling, USA), rabbit anti-P53 (2524S, Cell signaling, USA), rabbit anti-BAX (50,599, Cell signaling, USA), rabbit anti-Caspase-3 (A0214, ABclonal, $\mathrm{CN}$ ), mouse anti- $\beta$-actin (M1210, HuaAn, CN), and anti-GAPDHHRP (ET1702-66, HuaAn, CN). $\beta$-actin and GAPDH were used for the protein loading controls and analyzed with the Invitrogen iBright CL1500 Imaging System (Thermo Fisher Scientific, MA, USA). All determinations were repeated in triplicate.

\section{Histological characterization}

Tumor samples and adjacent normal tissues from patients were fixed in $10 \%$ neutral buffered formalin. Paraffin blocks were prepared and serial 5 - $\mu$ m-thick sections were cut from paraffin-embedded tumors by a Paraffin cutter (Leica, DE). The paraffin sections were deparaffinized and rehydrated for subsequent immunohistochemical staining against HCAR3 and INSL5. Following antigen retrieval, endogenous biotin activity was blocked using normal bovine serum and the sections were incubated with primary antibody in a humidified chamber. Horseradish peroxidase-conjugated secondary antibody was applied to the sections, followed by incubation with DAB (3,3'-diamino-benzidine) substrate for color development. The expression levels of the molecules were estimated by optical microscopy (OLMPUS, JP) and the images were captured using a Leica micro-imaging system (Lecia, DE).

\section{Statistical analysis}

All the experiments were performed independently in triplicate. These data points are presented using the mean \pm the standard errors of the mean (SEM). Statistical significance of experimental data was determined by a non-parametric test. Statistical analyses were performed using GraphPad Prism 8 (GraphPad Software Inc., USA). For all tests, significant differences were indicated using ${ }^{*} P=<0.05,{ }^{* * *} P<0.01$, or ${ }^{* * * *} P<0.001$.

\section{Results}

Microarray data characteristics and the identification of DEGs in CRC

The CRC expression microarray datasets GSE9348 and GSE110224 were calibrated and standardized, and the results are shown in Fig. 1a, d. When dataset GSE9348 was screened with the limma package (to identify genes with a corrected $P$ value $<0.05, \log$ FC $>2$ ), 700 DEGs were obtained. Among them, 454 genes with decreased expression and 246 genes with increased expression were identified. Overall, 286 DEGs were identified in the GSE110224 dataset, including 100 genes with increased expression and 186 genes with decreased expression. The differential expression of genes from the two sets of sample data included in each of the two microarrays are shown in Fig. 1b, e. The cluster heatmaps for the DEGs are shown in Fig. 1c, f. Further analysis found that the two independent datasets contained 207 common DEGs, including 64 genes that had increased expression and 143 with decreased expression in both the CRC datasets (Fig. 1g).

\section{GO and KEGG pathway enrichment analysis}

The common DEGs identified in the CRC datasets were further analyzed using a GO term and KEGG pathway enrichment analysis. The results of the KEGG enrichment pathway analysis showed that the signaling pathways enriched among the DEGs, included: bile secretion, IL-17 signaling pathway, rheumatoid arthritis, and PPAR signaling pathways (Fig. 2a). To understand the molecular basis, the DEGS were further categorized into molecular functions (MF), cellular component (CC), and biological process (BP) with a GO enrichment analysis. For the BPs, the common significantly enriched DEGs included organic anion transport and extracellular matrix organization (Fig. 2b). For CC, the common significantly enriched DEGs included extracellular matrix, apical part of cell, and collagen-containing extracellular matrix (Fig. 2c). For MF, the common enriched DEGs included receptor regulator activity, receptor ligand activity, and anion transmembrane transporter activity (Fig. 2d). Overall, these results indicated that the DEGs were mainly involved in extracellular matrix, extracellular matrix organization, organic anion transport, and receptor regulator activity. These are consistent with previous reports [12].

\section{Analyzing DEGs in colorectal cancer using a PPI network}

The aforementioned (207) colorectal cancer DEGs were analyzed using the STRING database to construct PPI networks. The data was imported into Cytoscape to calculate the topological characteristics of the network and 


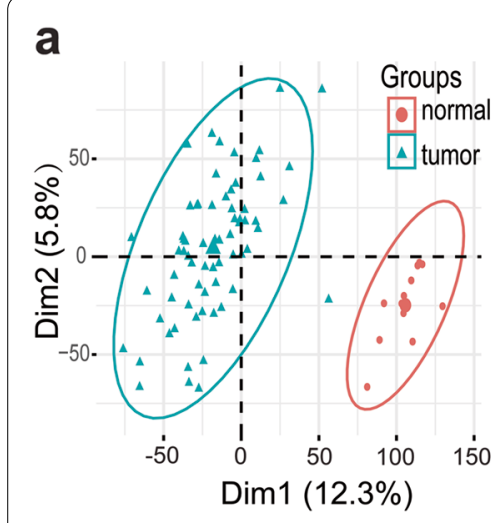

d

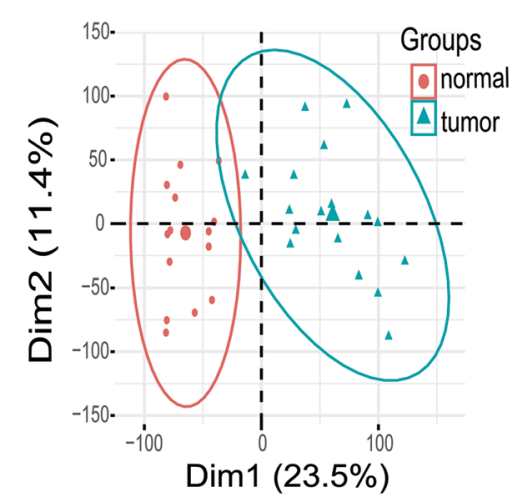

b

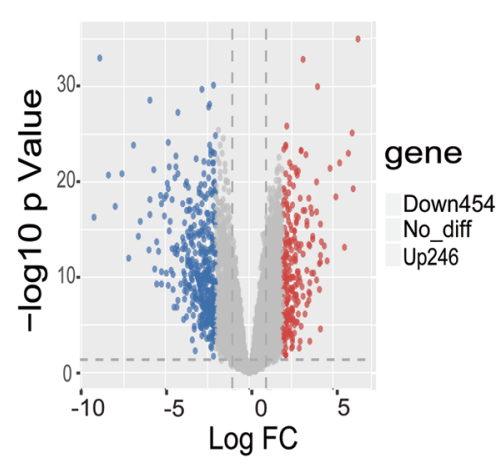

e

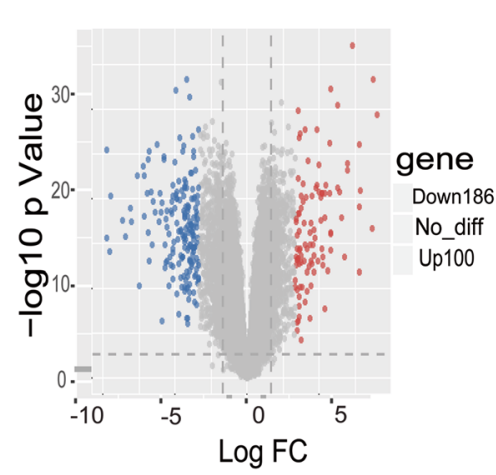

c

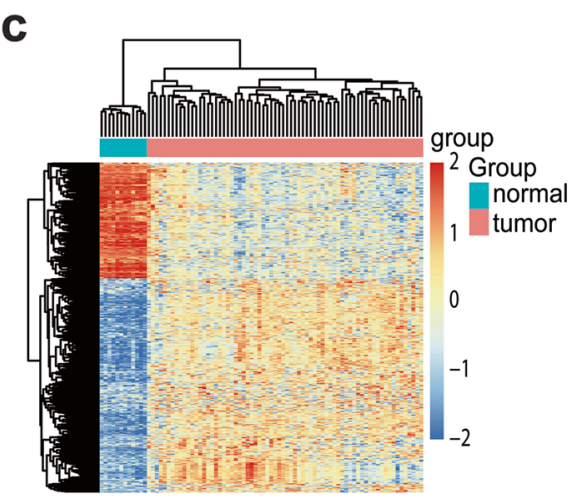

$\mathbf{f}$

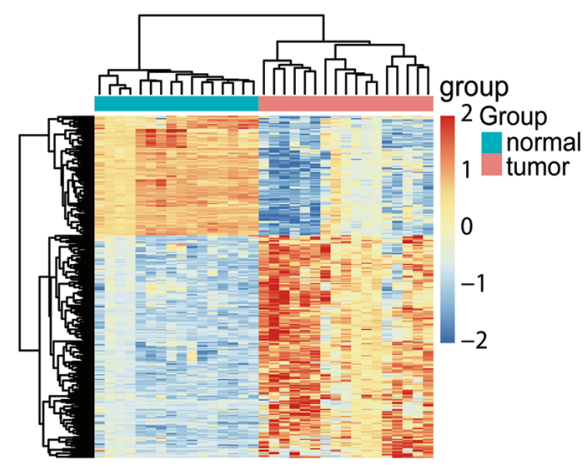

g

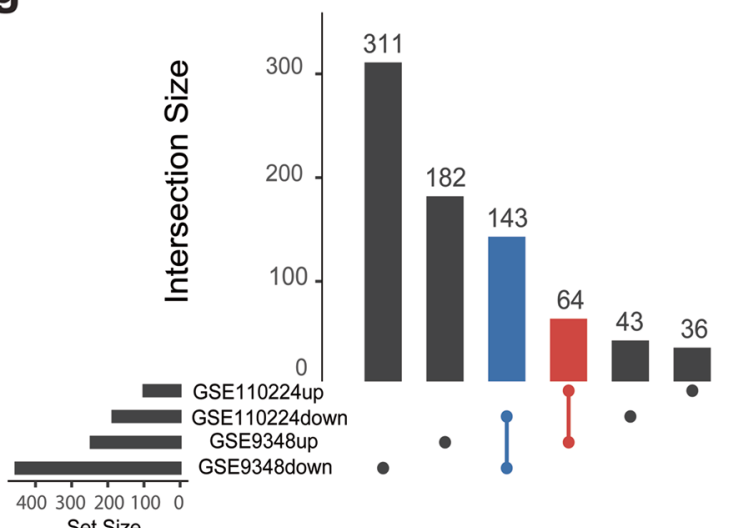

Fig. 1 Differential expression of data between two sets of samples. Principal component analysis plot of gene expression data showing the first two principal components in GSE9348 (a) and GSE11024 (d). Volcano plot of the differential expression of genes in GSE9348 (b). Principal component analysis plot of the gene expression data showing the first two principal components in GSE11024 (e), respectively. Hierarchical clustering heatmap for GSE9348 (c) and GSE1 1024 (f), DEGs screened on the basis of a fold change $>2.0$ and a corrected $P$ value of $<0.05$. (g) Upsetplot showing the number of DEGs in CRC tissues compared to that of normal colorectal tissues

determine each node. Six genes with increased expression and four genes with deceased expression in were selected as the hub genes, including CXCL1, HCAR3, CXCL6, CXCL8, CXCL2, CXCL5, PPY, SST, INSL5, and $N P Y 1 R$ (Fig. 2e, f). An additional network analysis was conducted to assess the interactions of some of the hub genes with selected competing endogenous RNAs (Figure S1).

A comprehensive literature search was carefully conducted and two novel core genes, HCAR3 and INSL5, had 


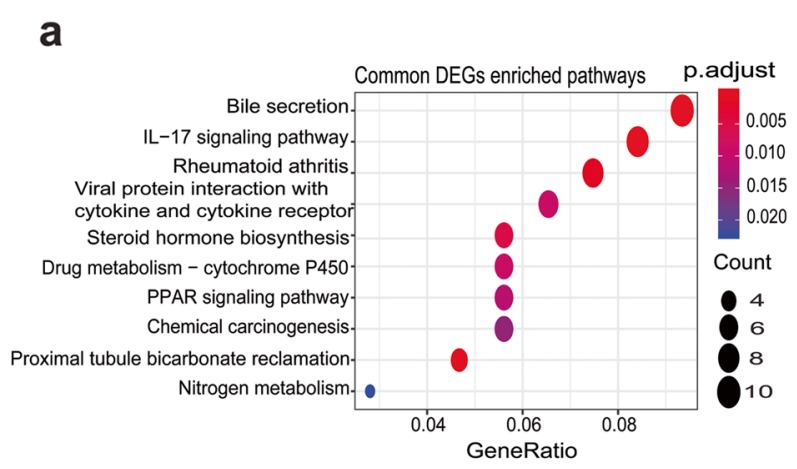

C

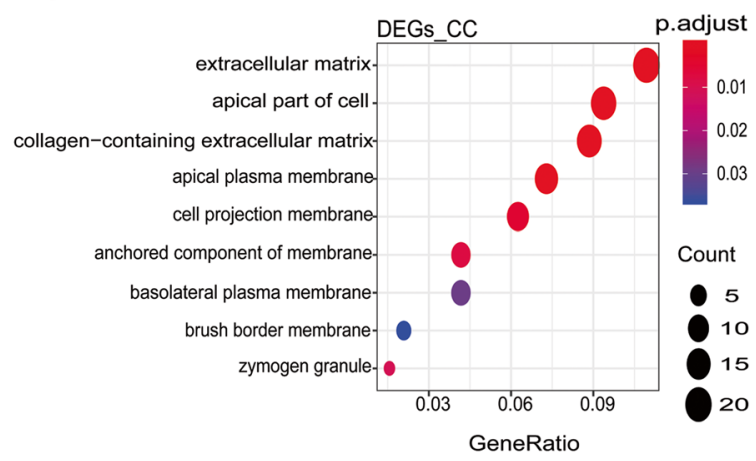

e

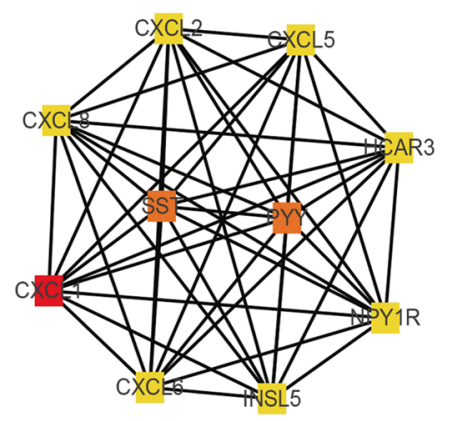

b

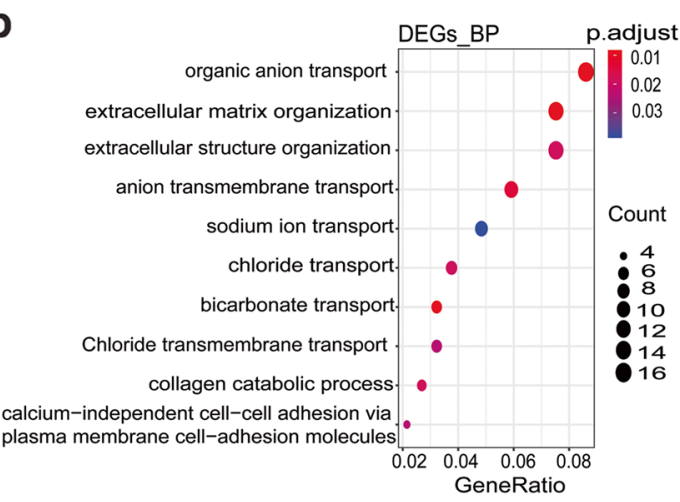

d

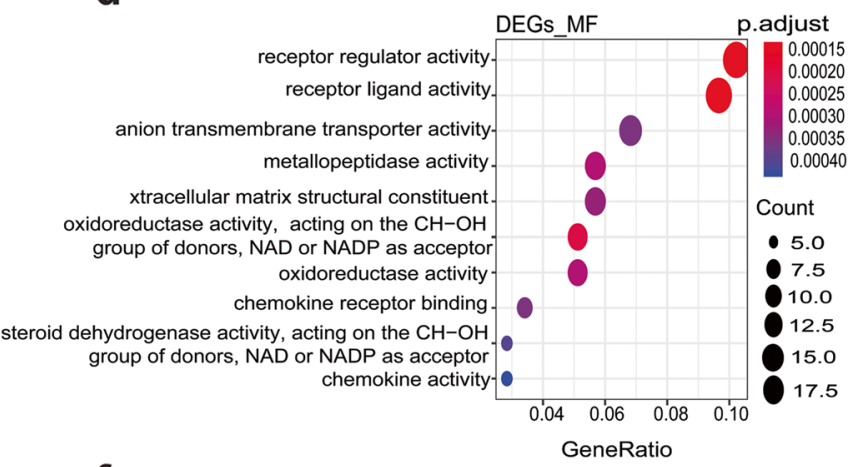

f

\begin{tabular}{|c|c|c|}
\hline Rank & Name & regulate \\
\hline 1 & CXCL1 & up \\
\hline 2 & PYY & down \\
\hline 2 & SST & down \\
\hline 4 & INSL5 & down \\
\hline 4 & HCAR3 & up \\
\hline 4 & NPY1R & down \\
\hline 4 & CXCL6 & up \\
\hline 4 & CXCL8 & up \\
\hline 4 & CXCL2 & up \\
\hline 4 & CXCL5 & up \\
\hline 4 & CXCL3 & up \\
\hline 4 & CXCL11 & up \\
\hline
\end{tabular}

Fig. 2 Integrated analysis of the common DEGs and the Protein-protein interaction (PPI) network. a KEGG pathways. b GO terms for biological processes. c GO terms for cellular components. $\mathbf{d}$ GO terms for molecular functions. e PPI network. f Hub genes

never previously been reported as being associated with CRC pathogenesis and progression.

\section{HCAR3 was downregulated in CRC associated with poor survival}

HCAR3 is a receptor for 3-OH-octanoid acid and a low affinity receptor for nicotinic acid, which mediates the negative feedback regulation of adipocyte lipolysis [13]. Previous studies reported that higher expression of HCAR3 was negatively correlated with survival time of cervical cancer patients [14]. In CRC patients, the average expression of HCAR3 was increased in CRC tissues compared to normal tissues (Fig. 3a). The expression of HCAR3 was positively correlated to survival time of CRC patients (Fig. 3b). HCAR3 expression changes were also detected with qPCR in five CRC patient samples. The RTPCR results showed that in patients 1, 3, and 5, HCAR3 was significantly increased in CRC tissues; in patient 2, there was no significant change between CRC tissue and normal tissue; whereas for patient 4, HCAR3 was significantly decreased in CRC tissues compared to the normal tissue (Fig. 3c). The protein level of HCAR3 in these 5 CRC samples was also evaluated using western blotting. However, there were no obvious protein level changes 


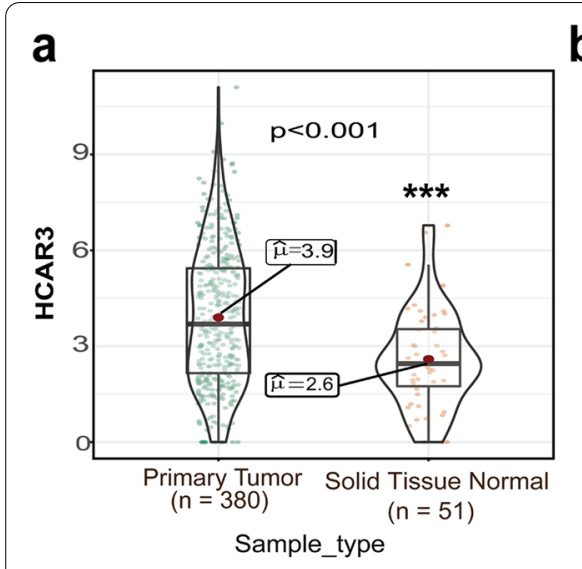

d

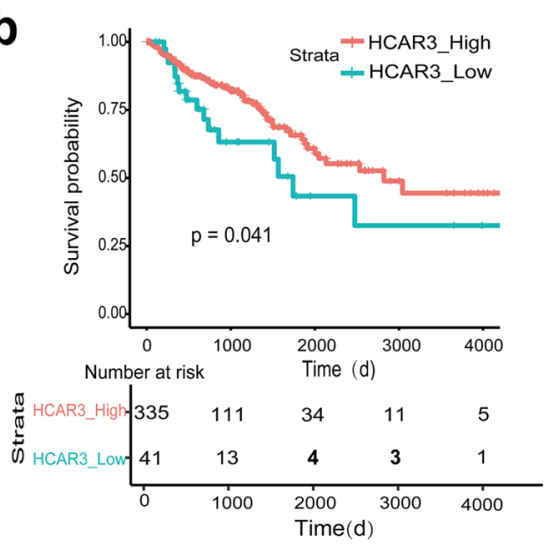

e

f

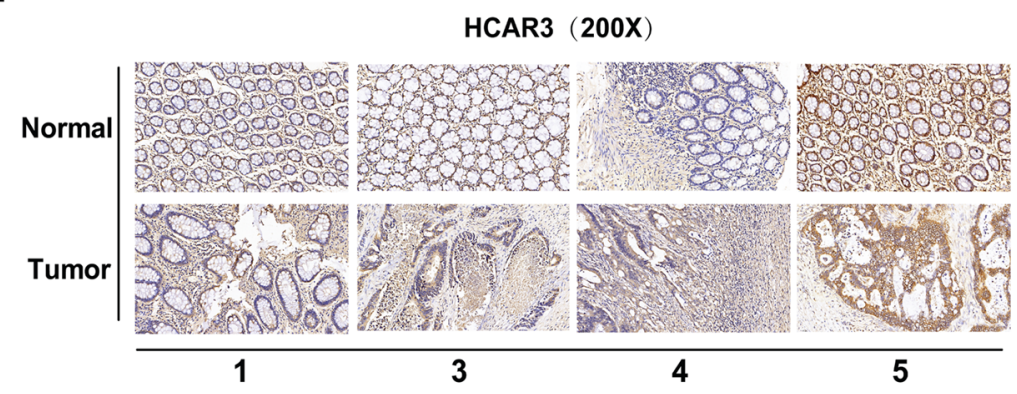

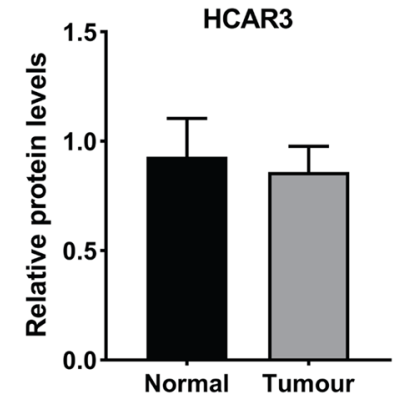

C

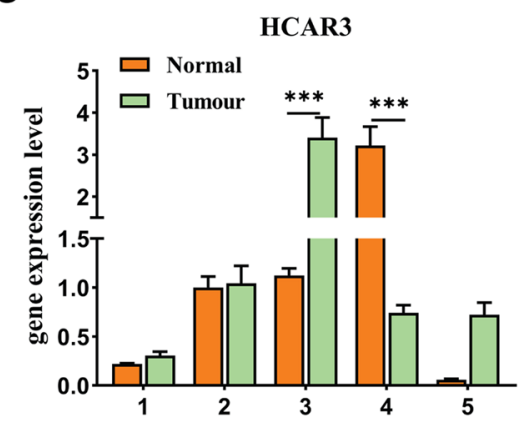

9

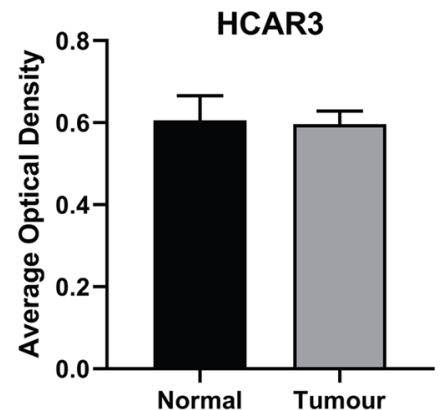

Fig. 3 The expression of HCAR3 in human colorectal cancer. aHCAR3 mRNA expression was significantly higher in colorectal cancer than in normal colorectal tissue samples $(* *, P<0.001)$. b Relationship between HCAR3 expression level and prognosis of patients with colorectal cancer $\left({ }^{*}, P=0.041\right)$. HCAR3 expression was detected in tumor tissues and adjacent normal tissues by RT-PCR $(\mathbf{c}), W B(\mathbf{d}, \mathbf{e})$, and $I H C(\mathbf{f}, \mathbf{g}) .{ }^{*}, P=<0.05 ;{ }^{* *}$, $P=<0.01 ; * *, P=<0.001$

for any of the samples (Fig. 3d, e). IHC assays were also performed for these samples, which were consistent with western blot results, and there was no significant HCAR3 protein signal increase compared to the adjacent nontumor tissues (Fig. 3f, g).

\section{High expression of INSL5 was associated with a better prognosis}

Based upon previous reports, higher expression of INSL5 promoted nasopharyngeal carcinoma (NPC) progression [15]. INSL5 belongs to the insulin superfamily [16] and may play a role in gut contractility and thymic development/regulation [17]. The expression of INSL5 was decreased in CRC tissue compared to normal tissue (Fig. 4a), and the expression of INSL5 was associated with a better prognosis for CRC patients (Fig. 4b). INSL5 expression status in CRC patient tissues was assayed with RT-PCR. INSL5 expression was significantly reduced in 4 samples, but not patient 3 (Fig. 4c). The protein level of INSL5 in these 5 CRC samples was also evaluated with western blotting. No obvious changes were detected for the INSL 5 protein bands (Fig. $4 \mathrm{~d}, \mathrm{e}$ ). IHC assays were also performed to show the endogenous INSL5 level in CRC tissues, but 


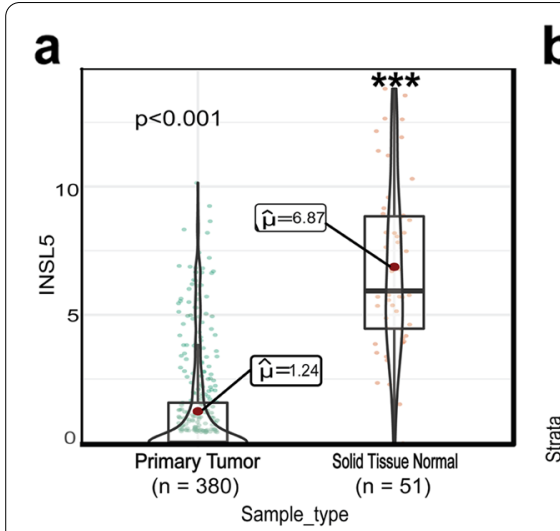

b

d

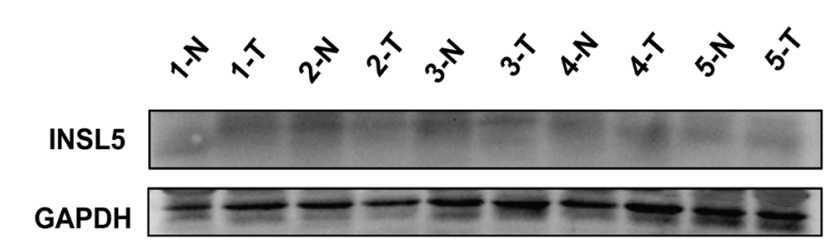

$\mathbf{f}$

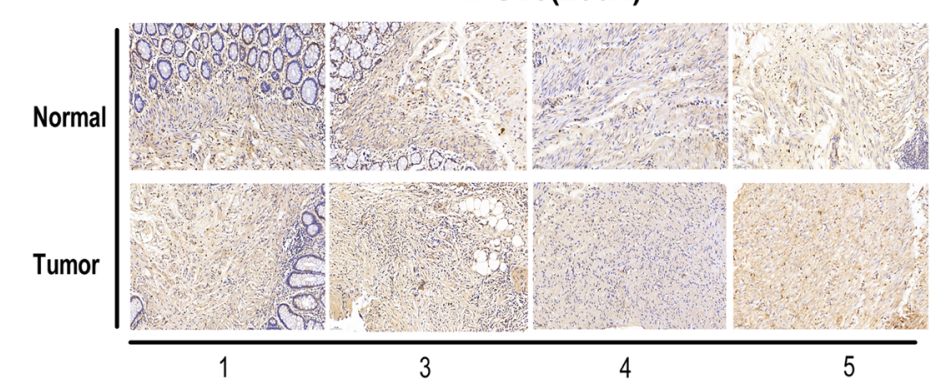

C

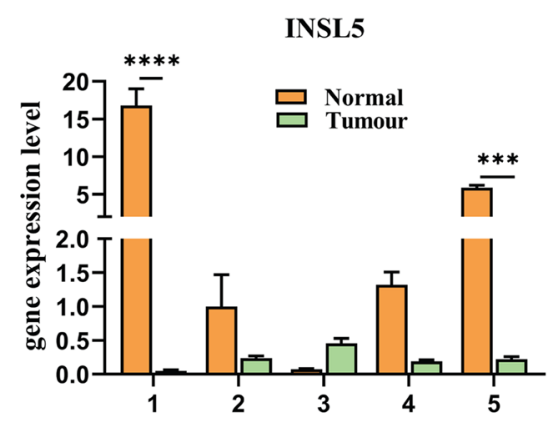

e

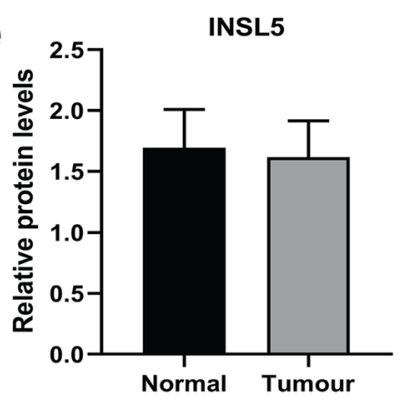

g

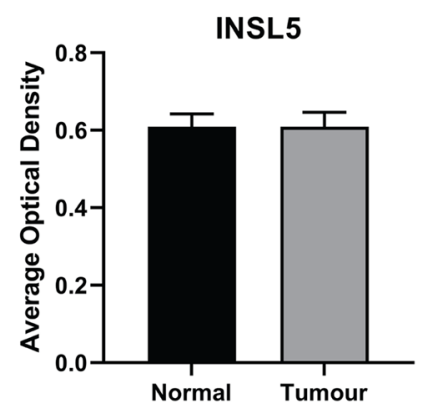

Fig. 4 The expression of INSL5 in human colorectal cancer. a Expression of INSL5 is reduced in colorectal cancer (***, $P<0.001)$. b The association of high-INSL5 expression with a good prognosis is highly statistically significant $(*, P=0.04)$. INSL5 expression was detected in tumor tissues and adjacent normal tissues by RT-PCR (c), WB (d, e), and IHC (f, g). ${ }^{*}, P=<0.05 ; * *, P=<0.01 ; * * *, P=<0.001$

no obvious protein signal changes were observed for all the detected samples (Fig. 4f, g).

INSL5 endogenous receptor is RXFP4, which belongs to GPCR family, also named GPCR142 [18]. INSL5 binding to RXFP4 results in inhibited cAMP accumulation [19]. Consistently, both INSL5 and RXFP4 are specifically and highly expressed in gastrointestinal tract. Therefore, the expression of RXFP4 in CRC tissue was also analyzed. Whilst the expression of RXFP4 was significantly lower in the tumor tissues $(p<0.001)$, the RXFP4 mRNA level was still maintained at a high level (Fig. 5a). The relationship between CRC prognosis and RXFP4 expression was also analyzed in TCGA database, where higher expression of RXFP4 was associated with better prognosis (Fig. 5b). The correlation between INSL5 and RXFP4 expression in CRC patients was determined to indicate whether the two genes were functionally relevant to prognosis. The best prognosis was observed for patients with high INSL5, as opposed to high expression levels of both INSL5 and RXFP4 (Fig. 5c). To further model this change, the expression of INSL5 and RXFP4 was determined in the CRC cell lines, of which HT29 and SW620 cells were selected to construct a stable overexpression system for INSL5 (Fig. 6a-c). CCK-8 assays demonstrated that overexpression of INSL5 significantly inhibited the proliferation of HT29 and SW620 cells (Fig. 6d, 


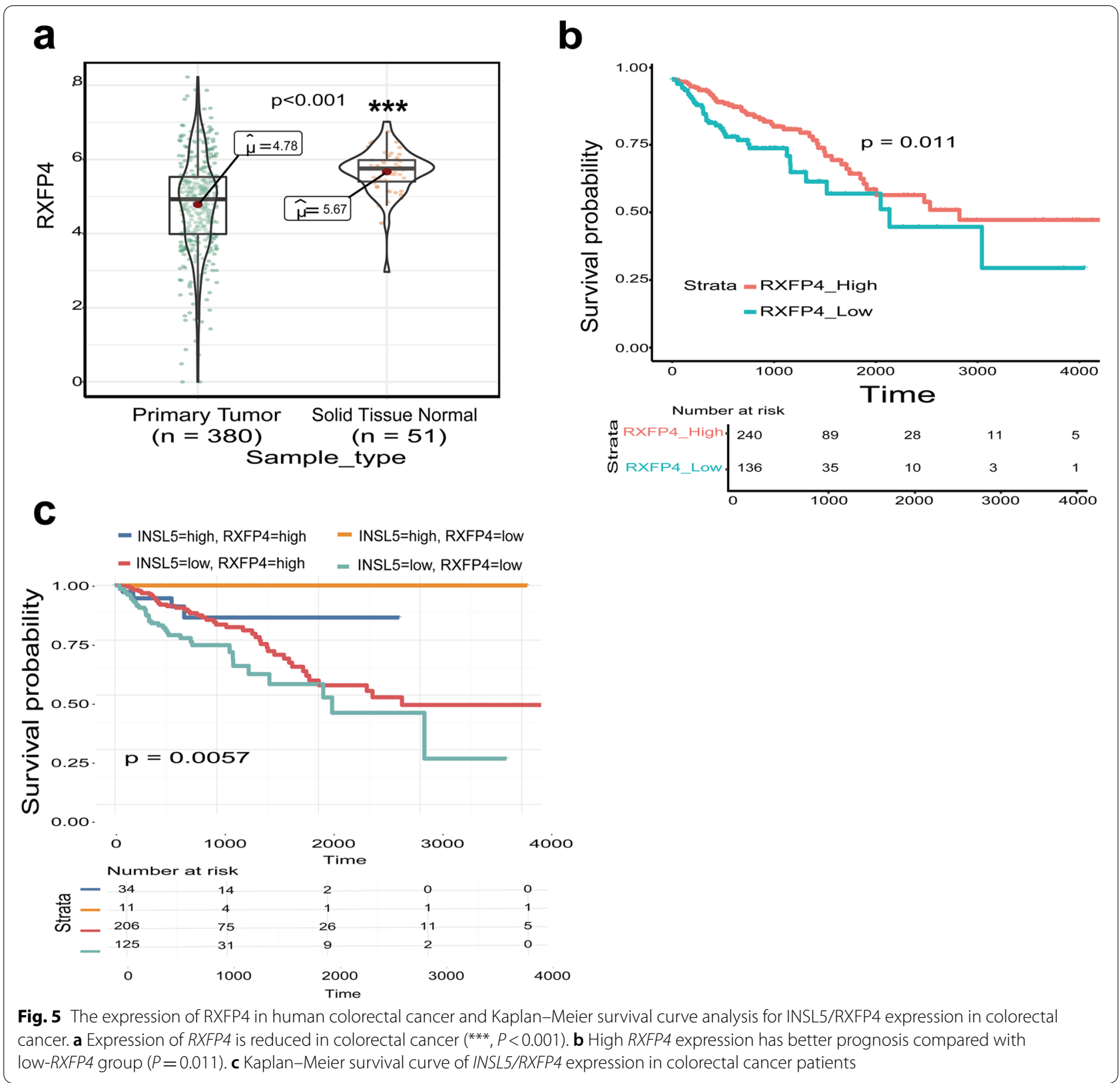

e). The result of cell cycle analysis found that overexpression of INSL5 did not significantly affect the cell cycle (Fig. 6f). Since the cells had the same mCherry fluorescence and PI detection channels, only FITC was used to indicate the total apoptosis of the cells (Fig. 6g). At the same time, the expression of the following apoptosis-related proteins was detected: Caspase 3, Cleaved-PARP, Bcl-2, BAX, P53 and P21. Except for Cleaved-PARP, the levels of the other proteins were not obviously different. Overexpression of INSL5 promoted the expression of Cleaved-PARP, which suggests that overexpression of INSL5 could promote the shearing of PARP (Fig. 6h, i).

\section{Discussion}

In this study, an integrated bioinformatics analysis was performed based upon the gene expression profiles of GSE9348 and GSE11024, which were obtained from the GEO database. The DEGs in CRC were analyzed using a GO term and KEGG signal pathway enrichment analysis. The major significantly enriched annotations were functions or pathways involved in extracellular matrix, 

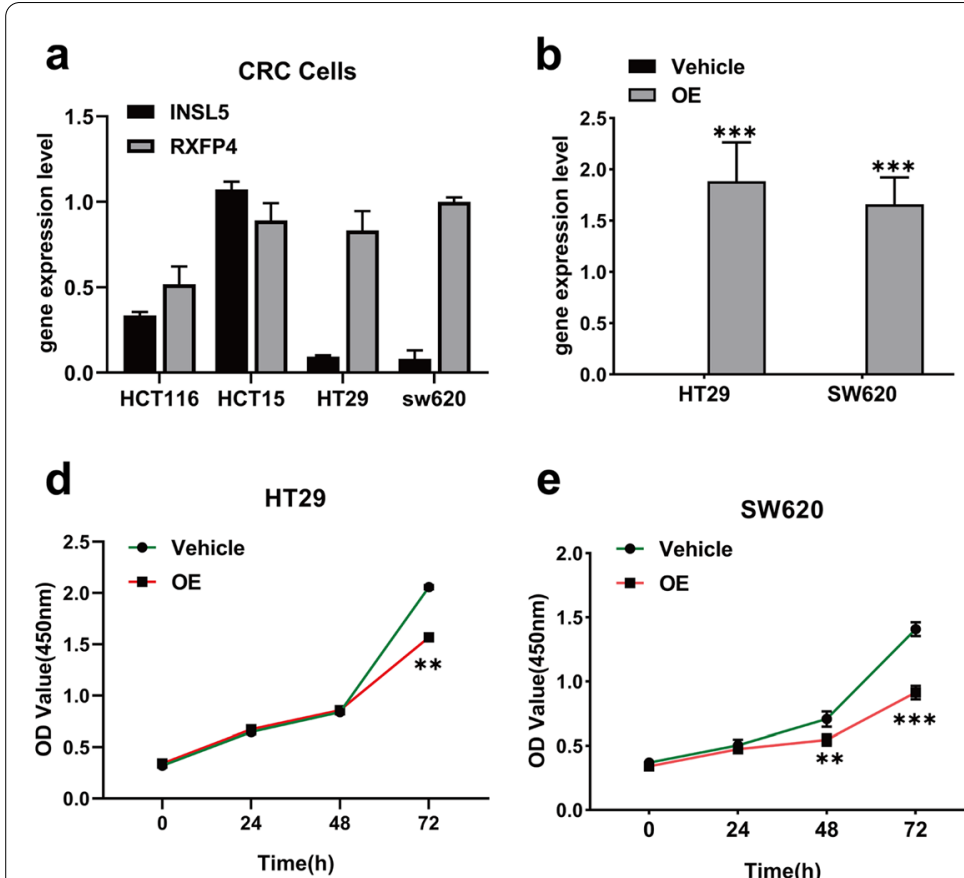

C
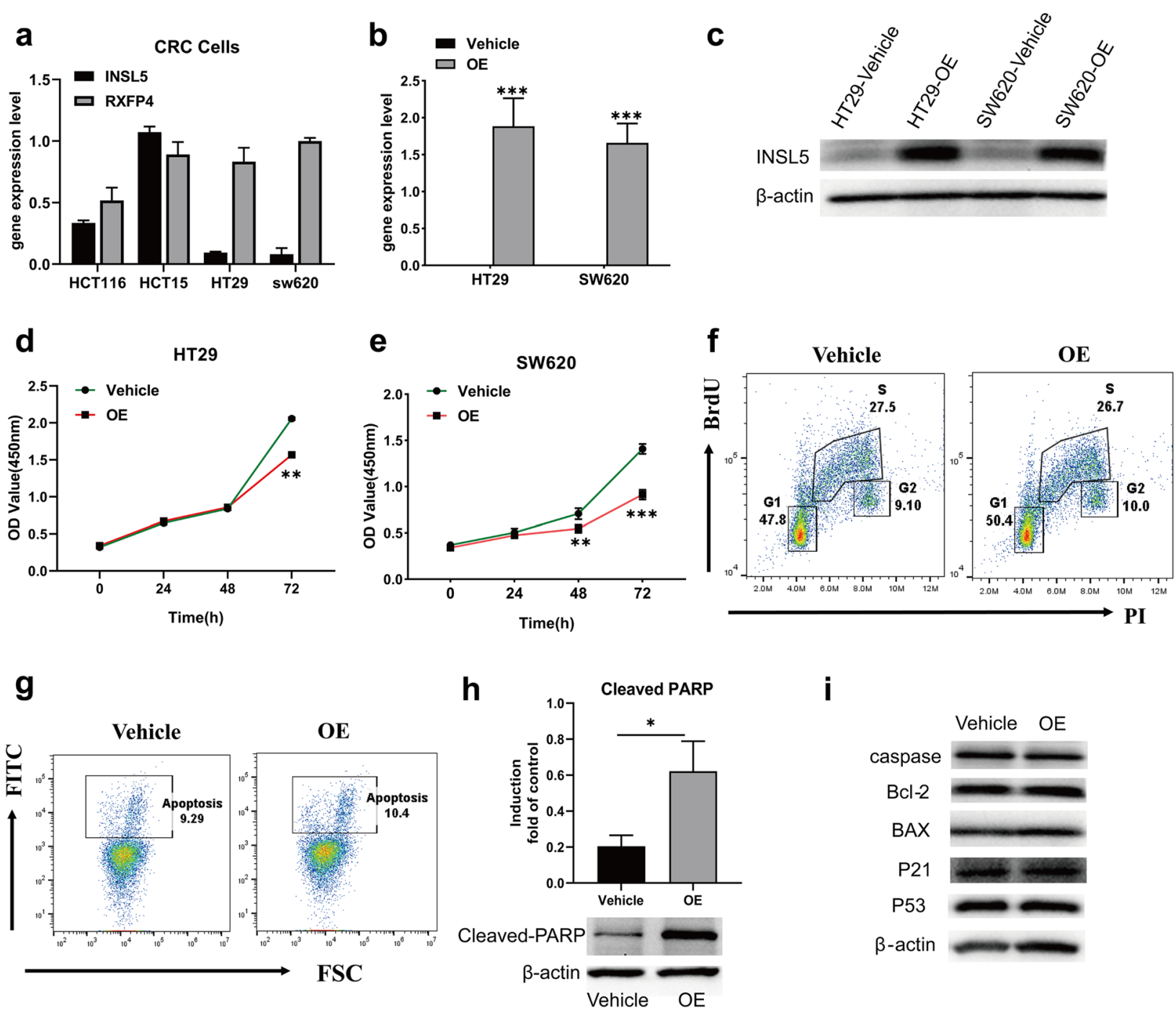

Fig. 6 Over-expression of INSL5 inhibited tumor cell proliferation. a Expression of INSL5 and RXFP4 in CRC cells. b, c Stable overexpression for INSL5 by retroviral transfection in HT29 and SW620 cells. d, e Results of CCK-8 assays indicated that INSL5 significantly inhibited proliferation. $\mathbf{f}$ Results of cell cycle in SW620. $\mathbf{g - i}$ Results of cell apoptosis in SW620. The experiments were repeated thrice and similar results were obtained. Representative data was shown. Each assay was performed at least in triplicate. ${ }^{*}, P=<0.05 ;{ }^{*}, P=<0.01 ;{ }^{* *}, P=<0.001$

extracellular matrix organization, organic anion transport, apical part of cell, and receptor regulator activity (Fig. 2). This analysis enabled the identification of 10 hub genes by constructing a PPI network analysis. Most of these had been reported in previous studies. Among these studies, the authors reported that CXCL1 and CXCL5 played roles in CRC progression and metastasis, and high expression levels of CXCL1 and CXCL5 were associated with poor prognosis in patients with CRC $[20,21]$. The CXC chemokine family appeared to have a complicated and multifaceted involvement in CRC. Tseng and Liu [22] have reported that lower expression of $P Y Y$ may relate to the development and progression of
CRC. SST is one of the cyclic tetradecapeptide hormones related to cancer growth, invasion, and metastasis [23]. A correlation between NPY1R and intestinal inflammation had previously been made, and NPY1R signaling was speculated to be a potential therapeutic target for colonic inflammation [24]. Two of the 10 hub genes, HCAR3 and INSL5, had not previously been found in prior studies $[20,21]$.

INSL5 is a member of the human insulin superfamily with an insulinotropic effect in colonic enteroendocrine $\mathrm{L}$ cells. As the endogenous ligand of RXFP4, INSL5 activates RXFP4 with high potency [25]. Mashima et al. [16] previously reported that INSL5 might be a unique 
marker of colorectal EECs and INSL5-RXFP4 signaling might play a role in an autocrine/paracrine fashion in colorectal epithelium and rectal neuroendocrine tumors. A recent study reported that INSL5 enhanced NPC progression and indicated that INSL5 might be a therapeutic target for NPC [15]. However, the study presented herein revealed that high expression of both INSL5 and RXFP4 was associated with a better prognosis for CRC (Fig. 4b and Fig. 5). However, the robust expression of RXFP4 in CRC indicated that its biological activity may not be greatly impaired. The differential expression of RXFP4 mRNA between the tumor and normal tissue may not be the major factor for CRC prognosis. This hypothesis was confirmed when CRC prognosis was further analyzed, which accounted for both INSL5 and RXFP4 expression. Indeed, the expression of INSL5 was the decisive factor for CRC prognosis, provided of course that RXFP4 was sufficiently expressed.

For the reasons stated above, a stable overexpression system for INSL5 in CRC cell lines was constructed, which enabled the finding that an overexpression of INSL5 can inhibit the proliferation of CRC cells (Fig. 6). The analysis of apoptosis pathway proteins revealed that overexpression of INSL5 can promote the cleavage of poly (ADP-ribose)-polymerase (PARP) (Fig. 6h, i). PARPs are a superfamily of multi-protein structures, which play an important role in DNA replication and DNA repair damage. Parthanatos is a cell death pathway that is not mediated by caspase [26]. It relies on PARP and mitochondrial-associated apoptosis-inducing factor (AIF). AIF nuclear translocation due to overactivation of PARP leads to large-scale DNA fragmentation and chromatin condensation, which leads to cell death and cell cycle arrest $[27,28]$. This may be the related regulatory mechanism through which INSL5 affects progression of colorectal cancer, further research may be warranted to understand the precise mechanism. Based upon these results, inhibition of INSL5 (or activation of RXFP4 with specific agonist) could be explored as a potential strategy for CRC treatment.

HCAR3 is a metabolite-sensing GPCR [29]. Consistent with gene profiling data, HCAR3 expression was higher in most CRC patient tissues, but the WB and IHC results showed no difference between tumor and its related adjacent tissues (Fig. 3). Moreover, no differences were observed among the CRC tissues. Kaplan-Meier survival curves showed that high expression of HCAR3 was significantly associated with better overall survival of CRC patients (Fig. 3b). Therefore, it is possible that the biological functions of HCAR3 might not be critical during CRC pathogenesis and progression. However, HCAR3 might be a potential biomarker for CRC prognosis. To test this hypothesis, the prognosis of CRC patients was stratified based upon the expression of INSL5 and HCAR3, in all but one case the prognosis was consistent with the expression of INSL5 and HCAR3 (Figure S2). This result indicated that HCAR3 could be used as a biomarker for CRC prognosis. However, further studies are needed to illuminate its roles in CRC tumorigenesis.

It is noteworthy that this study has some limitations, which can be addressed in future studies by expanding upon the limited tumor sample size, with additional samples to verify the results. Finally, more in vitro experiments and the addition of in vivo experiments could help enhance our understanding of the key functions of these genes in the context of CRC.

\section{Conclusions}

This integrated bioinformatics study presented 10 key hub genes associated with CRC, of which two were novel core genes. HCAR3 and INSL5 were expressed in tumor tissue and these were associated with poor survival and warrant further study with regard to their potential utility as CRC therapeutic targets.

\section{Abbreviations}

CRC: Colorectal cancer; DEGs: Differentially expressed genes; HCAR3: Hydroxycarboxylic acid receptor 3; INSL5: Insulin like 5; GEO: Gene Expression Omnibus; GO: Gene Ontology; KEGG: Kyoto Encyclopedia of Genes and Genomes; PPI: Protein-protein interaction; TCGA: The Cancer Genome Atlas; NPC: Nasopharyngeal carcinoma; RXFR4: Relaxin family peptide/ INSL5 receptor 4; GPCR: G protein-coupled receptor; PARP: Poly (ADP-ribose)-polymerase; AIF: Apoptosis-inducing factor.

\section{Supplementary Information}

The online version contains supplementary material available at https://doi. org/10.1186/s12957-021-02335-x.

Additional file 1: Table S1. RT-PCR Primer Sets [30-32].

Additional file 2: Figure $\mathbf{S 1}$ (a) Interaction networks for competing endogenous RNAs were produced as per the description in the supplementary information. The red rectangles indicate miRNA, green diamonds indicate IncRNA and purple circles represent mRNA. (b) UpSetR plot showing distribution of KEGG enrichment pathways for different genes. "Viral protein interaction with cytokine and cytokine receptor"; "Rheumatoid arthritis"; "L-17 signaling pathway"; "Bile secretion"; "Cytokine-cytokine receptor interaction".

Additional file 3: Figure S2 Kaplan-Meier survival curve of INSL5/HCAR3 expression in colorectal cancer patients.

\section{Acknowledgements}

We would like to thank Prof. Yiguo Hu for management, discussions, and support; Yunyu Fen for data analysis; and State Key Laboratory of Biotherapy, Sichuan University for their support.

\section{Authors' contributions}

ZW and BY led study design and provided experimental idea design. $X Y$ and WW carried out the experiments. XY and ZW performed data analysis and prepared the manuscript. LG and SQ assisted in tissue sample collection. ST provided data collection. All authors read and approved the final manuscript. 


\section{Funding}

The current work is funded by the National Natural Science Foundation of China (NO. 81860535) and Guizhou Innovation and Entrepreneurship Foundation for High-level Overseas Talent (No. [2019] 03).

\section{Availability of data and materials}

All data generated or analyzed during this study are included in this published article [and its supplementary information files]

\section{Declarations}

\section{Ethics approval and consent to participate}

Approval was obtained from the ethics committee of Tongren Municipal People's Hospital and written informed consent was obtained from each patient. The procedures used in this study adhere to the tenets of the Declaration of Helsinki.

\section{Consent for publication}

Not applicable.

\section{Competing interests}

The authors declare that they have no competing interests.

\section{Author details}

${ }^{1}$ Guizhou University Medical College, Guiyang 550025, Guizhou, China.

${ }^{2}$ Department of Oncology, Guizhou Provincial People's Hospital, Guizhou 550002 Guiyang, China. ${ }^{3}$ Tongren Municipal People's Hospital, Guizhou 554300 Tongren, China.

Received: 4 March 2021 Accepted: 16 July 2021

Published online: 21 August 2021

\section{References}

1. Sung H, Ferlay J, Siegel RL, Laversanne M, Soerjomataram I, Jemal A, Bray F. Global cancer statistics 2020: GLOBOCAN estimates of incidence and mortality worldwide for 36 cancers in 185 countries. CA Cancer J Clin. 2021;71:209-49.

2. Coppedè F, Lopomo A, Spisni R, Migliore L. Genetic and epigenetic biomarkers for diagnosis, prognosis and treatment of colorectal cancer. World J Gastroenterol. 2014;20:943-56.

3. Dekker E, Tanis PJ, Vleugels JLA, Kasi PM, Wallace MB. Colorectal cancer. Lancet. 2019;394:1467-80.

4. Siegel RL, Miller KD, Jemal A. Cancer statistics, 2019. CA Cancer J Clin. 2019;69:7-34.

5. Yiu AJ, Yiu CY. Biomarkers in colorectal cancer. Anticancer Res. 2016:36:1093-102.

6. Nobile MS, Cazzaniga P, Tangherloni A, Besozzi D. Graphics processing units in bioinformatics, computational biology and systems biology. Brief Bioinform. 2017;18:870-85.

7. Wang YR, Meng LB, Su F, Qiu Y, Shi JH, Xu X, Luo QF. Insights regarding novel biomarkers and the pathogenesis of primary colorectal carcinoma based on bioinformatic analysis. Comput Biol Chem. 2020:85:107229.

8. Xu H, Ma Y, Zhang J, Gu J, Jing X, Lu S, Fu S, Huo J. Identification and verification of core genes in colorectal cancer. Biomed Res Int. 2020;2020:8082697.

9. Zhou J, Xie Z, Cui P, Su Q, Zhang Y, Luo L, Li Z, Ye L, Liang H, Huang J. SLC1A1, SLC16A9, and CNTN3 are potential biomarkers for the occurrence of colorectal cancer. Biomed Res Int. 2020;2020:1204605.

10. Hou X, Hou N, Fu J, He X, Xiong H, Xie W, Jia G, Zuo X, Qin X, Pang M. Identification of key mRNAs and pathways in colorectal cancer. Nutr Cancer. 2021;73:1040-6.

11. Zhang Y, Wu W, Qu H. Integrated analysis of the gene expression changes during colorectal cancer progression by bioinformatic methods. J Comput Biol. 2019;26:1168-76.

12. Chen L, Lu D, Sun K, Xu Y, Hu P, Li X, Xu F. Identification of biomarkers associated with diagnosis and prognosis of colorectal cancer patients based on integrated bioinformatics analysis. Gene. 2019;692:119-25.
13. Ahmed K, Tunaru S, Langhans CD, Hanson J, Michalski CW, Kölker S, Jones PM, Okun JG, Offermanns S. Deorphanization of GPR109B as a receptor for the beta-oxidation intermediate $3-\mathrm{OH}$-octanoic acid and its role in the regulation of lipolysis. J Biol Chem. 2009;284:21928-33.

14. Ding $H$, Xiong XX, Fan GL, Yi YX, Chen YR, Wang JT, Zhang W. The new biomarker for cervical squamous cell carcinoma and endocervical adenocarcinoma (CESC) based on public database mining. Biomed Res Int. 2020;2020:5478574

15. Li SB, Liu YY, Yuan L, Ji MF, Zhang A, Li HY, Tang LQ, Fang SG, Zhang H, Xing S, Li MZ, Zhong Q, Lin SJ, Liu WL, Huang P, Zeng YX, Zheng YM, Ling ZQ, Sui JH, Zeng MS. Autocrine INSL5 promotes tumor progression and glycolysis via activation of STAT5 signaling. EMBO Mol Med. 2020;12:e12050.

16. Mashima H, Ohno H, Yamada Y, Sakai T, Ohnishi H. INSL5 may be a unique marker of colorectal endocrine cells and neuroendocrine tumors. Biochem Biophys Res Commun. 2013;432:586-92.

17. Belgi A, Hossain MA, Shabanpoor F, Chan L, Zhang S, Bathgate RA, Tregear GW, Wade JD. Structure and function relationship of murine insulin-like peptide 5 (INSL5): free C-terminus is essential for RXFP4 receptor binding and activation. Biochemistry. 2011;50:8352-61.

18. Liu C, Chen J, Sutton S, Roland B, Kuei C, Farmer N, Sillard R, Lovenberg TW. Identification of relaxin-3/INSL7 as a ligand for GPCR142. J Biol Chem. 2003;278:50765-70.

19. Liu C, Kuei C, Sutton S, Chen J, Bonaventure P, Wu J, Nepomuceno D, Kamme F, Tran DT, Zhu J, et al. INSL5 is a high affinity specific agonist for GPCR142 (GPR100). J Biol Chem. 2005;280:292-300.

20. Kawamura M, Toiyama Y, Tanaka K, Saigusa S, Okugawa Y, Hiro J, Uchida K, Mohri Y, Inoue Y, Kusunoki M. CXCL5, a promoter of cell proliferation, migration and invasion, is a novel serum prognostic marker in patients with colorectal cancer. Eur J Cancer. 2012:48:2244-51.

21. Zhuo C, Wu X, Li J, Hu D, Jian J, Chen C, Zheng X, Yang C. Chemokine (C-X-C motif) ligand 1 is associated with tumor progression and poor prognosis in patients with colorectal cancer. Biosci Rep. 2018;38:BSR20180580.

22. Tseng WW, Liu CD. Peptide $Y Y$ and cancer: current findings and potential clinical applications. Peptides. 2002;23:389-95.

23. Pyronnet S, Bousquet C, Najib S, Azar R, Laklai H, Susini C. Antitumor effects of somatostatin. Mol Cell Endocrinol. 2008;286:230-7.

24. Chandrasekharan B, Nezami BG, Srinivasan S. Emerging neuropeptide targets in inflammation: NPY and VIP. Am J Physiol Gastrointest Liver Physiol. 2013;304:G949-957.

25. Luo X, LiT, Zhu Y, Dai Y, Zhao J, Guo ZY, Wang MW. The insulinotrophic effect of insulin-like peptide 5 in vitro and in vivo. Biochem J. 2015;466:467-73.

26. Zhou Y, Liu L, Tao S, Yao Y, Wang Y, Wei Q, Shao A, Deng Y. Parthanatos and its associated components: promising therapeutic targets for cancer. Pharmacol Res. 2021;163:105299.

27. Fatokun AA, Dawson VL, Dawson TM. Parthanatos: mitochondriallinked mechanisms and therapeutic opportunities. Br J Pharmacol. 2014;171:2000-16.

28. Madungwe NB, Feng Y, Lie M, Tombo N, Liu L, Kaya F, Bopassa JC. Mitochondrial inner membrane protein (mitofilin) knockdown induces cell death by apoptosis via an AIF-PARP-dependent mechanism and cell cycle arrest. Am J Physiol Cell Physiol. 2018;315:C28-43.

29. Peters A, Krumbholz P, Jäger E, Heintz-Buschart A, Cakir MV, Rothemund S, Gaudl A, Ceglarek U, Schöneberg T, Stäubert C. Metabolites of lactic acid bacteria present in fermented foods are highly potent agonists of human hydroxycarboxylic acid receptor 3. PLoS Genet. 2019;15:e1008145.

30. Ru Y, Kechris KJ, Tabakoff B, Hoffman P, Radcliffe RA, Bowler R, Mahaffey S, Rossi S, Calin GA, Bemis L, Theodorescu D. The multiMiR R package and database: integration of microRNA-target interactions along with their disease and drug associations. Nucleic Acids Res. 2014;42:e133.

31. Shannon P, Markiel A, Ozier O, Baliga NS, Wang JT, Ramage D, Amin $\mathrm{N}$, Schwikowski B, Ideker T. Cytoscape: a software environment for integrated models of biomolecular interaction networks. Genome Res. 2003:13:2498-504.

32. Lex A, Gehlenborg N, Strobelt H, Vuillemot R, Pfister H. UpSet: Visualization of Intersecting Sets. IEEE Trans Vis Comput Graph. 2014;20:1983-92.

\section{Publisher's Note}

Springer Nature remains neutral with regard to jurisdictional claims in published maps and institutional affiliations. 